\title{
When Reconstruction Comes Around: Ni, Cu, and Au adatoms on $\delta$-MoC(001)
}

Gian Giacomo Asara, ${ }^{\mathrm{a}, \mathrm{b}}$ Francesc Viñes, ${ }^{\mathrm{b}}$ Josep M. Ricart ${ }^{\mathrm{a}}$, José A. Rodriguez ${ }^{\mathrm{c}}$ and Francesc Illas ${ }^{\mathrm{b}}$

a) Departament de Química Física i Inorgànica, Universitat Rovira i Virgili, C/ Marcel·lí Domingo s/n, 43007 Tarragona, Spain

b) Departament de Química Física \& IQTCUB, Universitat de Barcelona, C/Martí i Franquès 1, 08028 Barcelona, Spain

c) Chemistry Department, Brookhaven National Laboratory, Upton, New York 11973, United States

\begin{abstract}
The interaction of $\mathrm{Au}, \mathrm{Cu}$, and $\mathrm{Ni}$ metal atoms with the $\delta$-MoC(001) surface was studied using periodic Density Functional $(D F)$ calculations to analyse adsorption energies and equilibrium geometries, work functions, atomic charges, Projected Density Of States (PDOS), and shifts of the transition metal $d$-band center. The atomic adsorption is found to cause an in-plane distortion of the surface, and, besides, the interaction strength turns to be coverage dependent. A lower coverage allows for a better accommodation of the adsorbate, alongside causing a $d$-band centre shift to more negative energies, as shown by plots of the PDOS. Regardless of the coverage, interaction strength diminishes following the order $\mathrm{Ni}>\mathrm{Cu}>\mathrm{Au}$. Adatom chemical activity can be inferred based on the calculated $d$-band centre; $\mathrm{Ni}$ being the most active metal, followed by $\mathrm{Cu}$, and $\mathrm{Au}$ later on for every coverage studied. This result correlates well with experiments on other transition metal carbides. Atomic adsorption also notably diminishes surface work function, with the concomitant repercussions on the electrocatalysis field.
\end{abstract}




\section{Introduction}

The manifold technological applications of Transition Metal Carbides (TMC) are nowadays well-known, and they have been the subject of several reviews in the last years [1,2]. For instance, such materials are currently widely used as cutting tools or covering materials, due to their extreme hardness. This feature and other important physical properties like their high electric conductivities and refractory melting temperatures have been interpreted by means of the analysis of the covalent, metallic, and ionic contributions to the chemical bonding between the transition metals and the carbon atoms [3]. From the early seventies, some other promising and more exciting possible uses of TMC captured the attention of the scientific community, including their usage as heterogeneous catalysts for industrial reactions: High activities have been found for both TMC single crystals and nanometric powders. In some cases, the TMC catalytic activity has been estimated to be higher than currently used industrial catalysts, as happened for the water gas shift reaction, where Density Functional $(D F)$ calculations predicted that $\mathrm{TiC}(001)$ surface overtake the industrial $\mathrm{Cu}$-based catalyst [4]. In the field of heterogeneous catalysis models, TMC have been explored as well as non-innocent supports for noble and coinage metal catalysts, but also as electrocatalysts in fuel cells [5]. For instance, metal clusters supported on TMC have been used for desulfurization $\left(\mathrm{DeSO}_{\mathrm{x}}\right)$ reactions [6], the hydrogenation of $\mathrm{CO}_{2}$ to obtain methanol or methane [7], and also for oxidation reactions $[8,9]$.

From earlier theoretical works it is found that the carbide-metal adatom interaction induces an important charge polarization in the latter [10-13], partially reducing the admetal and consequently boosting the activation of small organic or inorganic molecules adsorbed on the system [6-789,14-19]. Polarization of the electron density results in a charge accumulation in the proximity of the adsorbed transition metals, which is considered the key aspect in the enhanced catalytic activity of TMC-supported metal clusters [10]. This background is capitally important when designing new catalysts combining transition metals with TMC, together with the linear relation demonstrated among adsorption and activation energies [20]. In many cases simple 
computational models well-reproduced in silico experimental trends for adsorption energies, adsorbate geometries, and preferred adsorption sites, recovering the experimental data on the activity trends of various TMC-metal nanoparticles composite systems.

In this work we present a detailed analysis of the adsorption of single $\mathrm{Au}, \mathrm{Cu}$, and $\mathrm{Ni}$ atoms on the (001) surface of $\delta$-MoC as carried out previously on a few metal-TMC combinations [10-13]. The choice of this transition metals is also motivated by recent theoretical and experimental work on $\mathrm{CO}_{2}$ hydrogenation to methanol, catalysed precisely by $\mathrm{Au}, \mathrm{Cu}$ and $\mathrm{Ni}$ supported on $\mathrm{TiC}(001)$ [6,19]. Molybdenum carbides display interesting catalytic properties [2] which could be enhanced by the deposition of metals such as $\mathrm{Ni}, \mathrm{Cu}$ or $\mathrm{Au}$ [1]. Here it will be shown that surface reconstruction and in-plane relaxation happen to be determining when supporting metals on $\delta$ $\operatorname{MoC}(001)$. In addition, adsorption energy trends and changes in surface properties such as work function or atomic charges have been identified, and connected to the most stable adsorption configurations. The single atom adsorption provides a first idea of the interactions of a given metal with the carbide surface and, subsequently, how the support can affect the adatoms in a larger cluster.

\section{Computational details}

Adsorption of $\mathrm{Cu}, \mathrm{Ni}$, and $\mathrm{Au}$ metal atoms has been studied using standard state-of-the-art periodic DF calculations. Four-layered slabs of $\delta$-MoC were cut from the bulk perpendicular to the [001] direction and later on optimized. Atoms included in the uppermost 2 layers plus the adatom were fully allowed to relax whereas the two bottom layers were kept fixed at the previously optimised bulk positions [21]. Interaction between translationally-repeated slabs was avoided adding $10 \AA$ of vacuum along the [001] direction. Top image of the pristine relaxed surface is shown in Figure 1.

Three different slab models have been used to represent diverse coverages. The smallest cell is a $(\sqrt{ } 2 \times \sqrt{2}) R 45^{\circ}$ supercell exposing two Mo and two $C$ surface atoms in a square arrangement, see Figure 1. This latter cell has been re-baptised $(1 \times 1)$ for simplicity. The other two employed 
supercells for each surface are - following the above notation- $(2 \times 2)$ and $(3 \times 3)$ supercells. These permits one to scan metal coverages $(\theta)$ from 0.25 to 0.028 . Following a previous computational strategy [21], we used the Perdew-Burke-Ernzerhof ( $P B E)$ exchange-correlation functional [22], given its suitability in describing Molybdenum carbides. Calculations were carried out using the VASP computational package [23]. All calculations were carried out in a spin-polarized fashion. Valence electrons have been described using a plane-wave basis set with an associated maximum kinetic energy of $415 \mathrm{eV}$. The effect of the core electrons on the valence electron density was described using the Projector Augmented Wave (PAW) method [24]. The electron density was considered converged when subsequent energy minimisation steps lead to a maximum difference of $10^{-6} \mathrm{eV}$. Similarly, structures where optimised until forces calculated on each relaxed atom where smaller than $0.02 \mathrm{eV} \AA^{-1}$. For an accurate evaluation of the total energy of the system a MonkhorstPack k-point grid of $5 \times 5 \times 1$ was used for the sampling of the first Brillouin zone on all the employed supercells.

The adsorption energy has been calculated as:

$$
E_{a d s}=E_{M / T M C}-E_{M}-E_{T M C}
$$

where $E_{a d s}$ is the adsorption energy, $E_{M / T M C}$ is the energy of the supercell with the metal adatom, $E_{M}$ is the energy of the metal atom in the vacuum, and, finally, $E_{T M C}$ is the energy of the pristine carbide slab. Within this formalism, the more negative the $E_{a d s}$ values, the stronger the interaction. All stable structures have been identified as minima in the potential energy surface by numerical calculation of the Hessian matrix, obtained by finite displacements of the adatom coordinates. Vacuum atomic energy references were gained by placing the metal atom in a large $9 \times 10 \times 11 \AA$ asymmetric box and calculating the total energy. Atomic charges for adatoms were estimated applying a Bader analysis [25]. The surface work function was calculated by subtracting the Fermi energy value of the system under study from the electron potential value in the vacuum region. The centre of the $d$-band was obtained by plotting the Projected Density of States (PDOS) and numerically integrating the density in the $d$-band region of the total Density Of States (DOS). 


\section{Results}

Let us begin with the clean $\delta$-MoC(001) surface. One of its main features is the surface rumpling, a process mostly concerning the atoms of the outermost layer of a surface. Atomic relaxation changes the inter-planar distance along the axis perpendicular to the surface, to a different extent for anions and cations. This process has been intensively studied in oxides [26], in the (001) surface of rock-salt materials such as sodium halides [27], and it is found also in the (001) surface of early transition metals carbides [3]. For carbides, it implies an inward relaxation of the metal atoms at the surface, together with a displacement of the carbon atoms towards the vacuum. It is noteworthy that $\delta$-MoC is the TMC where this effect is most pronounced. DFT calculations using PW91 [28] functional predicted an intralayer rumpling displacement of $0.24 \AA(0.26 \AA$ calculated at RPBE level) [3]. Recent calculations performed with the PBE functional back up both results, with a calculated value of $0.25 \AA$. However, such relaxation does not imply any reconstruction, that is, the relative in-plane positions of the atoms and long range periodicity do not change. As it will be shown below, an important reconstruction occurs as consequence of metal atoms adsorption.

Along this line we studied the adsorption of $\mathrm{Ni}, \mathrm{Cu}$, and $\mathrm{Au}$ atoms on the relaxed $\delta$ MoC(001) surface. Due to its surface symmetry, see Figure 1, six highly-symmetric adsorption sites have been explored, see previous studies for their definition and notation [29]. However, for these metals only two particular sites happen to be energetically competitive: The three-fold hollow site neighbouring two surface Mo atoms and one surface $\mathrm{C}$ atom (MMC), and a four-fold hollow site, where metal adatoms are simultaneously bridging two Mo and two $\mathrm{C}$ surface atoms (Hollow). Energetic, geometric, and estimations of surface-related properties are encompassed in Table 1, connected to the most stable sites found for each particular case.

It is noteworthy that $\mathrm{Au}$ atoms clearly prefer to sit on MMC sites regardless of the coverage, and so it happens for $\mathrm{Ni}$ atoms, yet on the Hollow sites. At the fringe of both situations we find $\mathrm{Cu}$, whose site occupation is a function of the coverage: At $\theta=0.028 \mathrm{Cu}$ clearly prefers to sit on Hollow sites, and so does at medium coverage $(\theta=0.0625)$, although at this latter coverage the MMC sites 
get closer in energy, with an $E_{a d s}$ of $-2.75 \mathrm{eV}$, i.e. only $0.14 \mathrm{eV}$ destabilized with respect the Hollow site. At the largest coverage explored $(\theta=0.25)$ the difference in energy between both sites is only $0.04 \mathrm{eV}$, and so they are considered isoenergetic situations, although further discussion centres on the Hollow site, in order to get site-independent trends.

It is worth to highlight that, regardless of the coverage and nature of the adsorbed atom, a significant in-plane reconstruction of the $\delta$-MoC surface atoms is observed, quantified in an observed expansion of the hollow area $(A)$, see Table 1 and sketch in Figure 2 for a practical definition. Generally speaking, the adsorption of these metal atoms provokes a separation of surface Mo atoms involved in the adatom coordination, yet also on the surface $\mathrm{C}$ atoms, but to a lesser extent. As observed in Table 1 and Figure 2, the atomic adsorption increases $A$ up to $\sim 25 \%$ at the lowest coverage treated. This adsorption-triggered deformation also involves neighbouring unoccupied hollow sites, where such effect is present but more diluted. However, this mediumrange perturbation is at the origin of the lower surface deformation found at $(\theta=0.25)$, given that relaxations cancel with opposite forces of vicinal images. In any case, the stability of $\mathrm{Ni}, \mathrm{Cu}$ and $\mathrm{Au}$ metal adatoms on $\delta$-MoC $(001)$ is biased by the surface deformation, as shown in Figure 2 . The larger the allowed deformation, the stronger the bonding to the carbide surface.

One consequence of this is that the in-plane relaxation allows the adatoms to sensibly decrease the height $(h)$ from the $\delta$-MoC(001) surface, see Table 1, in perfect line with the featured stronger bonding. Nevertheless, note that the adsorption strength is also affected by the lateral repulsions between adatoms. To this end Bader charges $(Q)$ have been obtained for the adsorbed atoms, and shown in Table 1. Regarding Au adsorption, calculated Bader charges are always negative, implying a $\mathrm{MoC} \rightarrow \mathrm{Au}$ charge transfer, as found for other TMCs [10]. The amount of charge transfer slightly increases when decreasing the coverage, or, viewed from another perspective, the charge transfer is hindered at large coverages, given the repulsion of close negatively-charged Au adatoms. Thus, at lowest coverage Au adatoms are more negatively-charged. This feature is kept for $\mathrm{Ni}$ and $\mathrm{Cu}$ situations, although here the charge transfer is adatom $\rightarrow \mathrm{MoC}$, 
with an increase of such transfer when increasing the coverage, a fact that allows stating that electron-electron repulsion is governing lateral interactions of the metal adatoms.

The surface charge rearrangement is largely affecting the computed work functions. Indeed, atomic adsorption seems to always dramatically lower the work function of the $\delta$-MoC(001) surface, from previous estimations of $\sim 7.5 \mathrm{eV}$ for the naked surface [21] to present values ranging 3.4-4 eV, a feature that goes along with the above-commented electron density depletion when increasing the metal coverage. The large variation of the work function induced by the presence of the adsorbate may seem counter intuitive since one normally relates positively/negatively charged adsorbates to decrease/increase the surface work function. However, it must be pointed out that these general trends are normally encountered in metallic surfaces with a highly polarizable conduction band whereas transition metal carbides have large covalent and ionic bonding contributions. Moreover, even in the case of metallic surfaces it is not always possible to relate the adsorbate charge with the change of work function. This was first pointed out by Petterson and Bagus for $\mathrm{Cl}$ on $\mathrm{Cu}(100)$ [30] and has been later discussed by Michaelides et al. [31] who have shown that a work function decrease induced by $\mathrm{N}$ adsorption on $\mathrm{W}(100)$ is consistent with a negative charge on N. In a more systematic work, Migani et al. [32] have also shown that the change on the surface work function induced by the presence of adsorbed chloride can be either positive or negative, depending on the surface metal. A recent analysis by Bagus et al. [33] extends these arguments to the case of $\mathrm{I}$ on $\mathrm{Cu}(111)$ and shows that negatively charged adsorbates can result in a decrease or increase of the surface work function. In any case, these simple charge redistributions arguments helps in the understanding of the promising properties of transition metal carbides as supports for noble metal atoms in electrochemical catalytic applications [5], but also extends and recovers recent results obtained on $\mathrm{Mo}_{2} \mathrm{C}$ [34].

Last but not least, the adsorption energy trends are dependent on the coverage (and degree of surface reconstruction), but also, for any coverage, the bonding strengths decrease in the order $E_{a d s}(\mathrm{Ni})>E_{a d s}(\mathrm{Cu})>E_{a d s}(\mathrm{Au})$. This trend seems to be intimately connected with the generated $d$ - 
band centre of the adsorbed metal atoms, as extracted from PDOS shown in Figure 3. For any of the studied metals the $d$-states shift to more negative energies when decreasing the coverage, coherent with a stronger interaction. Comparing the $d$-band centre for the different metals, an approximated ranking of their catalytic activity can be extracted, as for every coverage studied the activity would decrease in the order $\mathrm{Ni}>\mathrm{Cu}>\mathrm{Au}$, given that $d$-band centres are shifted towards Fermi level in the same order, i.e. $d$-band centre of $\mathrm{Ni}$ is the least negative, and that of $\mathrm{Au}$, the most. This last relation has been confirmed in ongoing experiments for the activation of methane on $\mathrm{M} / \mathrm{MoC}$ surfaces $(\mathrm{M}=$ $\mathrm{Ni}, \mathrm{Cu}$ or $\mathrm{Au}$ ) [35] and such trend also agrees with data for $\mathrm{CO}_{2}$ hydrogenation on $\mathrm{M} / \mathrm{TiC}(001)$ [7].

The present results for the interaction of $\mathrm{Au}, \mathrm{Cu}$, and $\mathrm{Ni}$ with the $\delta$-MoC(001) surface seem to point towards an intrinsic instability of the surface or to an intrinsic high reactivity. This is in agreement with experimental evidence that, up to now, it has not been possible to prepare a welldefined $\delta$-MoC(001) surface. All experiments lead to polycrystalline samples, often with mixed MoC phases [36], which, in any case, include a non-negligible part of $\delta$-MoC(001) surfaces. The shifts in the position of the $\mathrm{C}$ atoms of $\delta$-MoC(001) induced by the admetals are important due to the fact that these $\mathrm{C}$ atoms are not simple spectators in the presence of C-containing adsorbates $[7,36,33]$. For instance they can make bonds with molecules such as $\mathrm{CO}$ or $\mathrm{CO}_{2}$ and $\mathrm{CH}_{\mathrm{x}}$ groups generated during the reforming of hydrocarbons $[2,7,36,37]$.

\section{Conclusions}

A systematic DF study of the interaction between $\mathrm{Ni}, \mathrm{Cu}$ and $\mathrm{Au}$ atoms and the non-polar (001) surface of $\delta$-MoC has been performed at different coverage $\theta$ ranging values between 0.028 and 0.25 , and analysing a variety of surface properties such adsorption energies, equilibrium geometries, work functions, atomic charges, partial density of states and shifts of the transition metal $d$-band centre have been analysed.

$\mathrm{Au}(\mathrm{Ni})$ atoms show a clear preference for MMC (Hollow) sites at any of the considered coverage, whereas $\mathrm{Cu}$ prefers to adsorb on Hollow sites at low coverage while at MMC sites at high coverage. At medium coverage $\mathrm{MMC}$ and Hollow geometries for $\mathrm{Cu}$ are essentially considered 
isoenergetic.

$\mathrm{Ni}, \mathrm{Cu}$ and $\mathrm{Au}$ atomic adsorption always causes a large in-plane deformation of the surface with an increment of up to $\sim 25 \%$ of the underlying Hollow area, also affecting neighbouring hollow sites, although to a lesser extent. The ability to accommodate the adatom turns to be a function of $\theta$, and increasing the coverage causes a reduction of the strength of the adsorbate-surface interaction.

An analysis of the PDOS shows how a higher atomic coverage produces a shift of the $d$ band to more positive energies, i.e. closer to Fermi level, in line with a decrease of the adsorption energy calculated. For every coverage, the calculated $d$-band centre suggests that activity decreases in the order $\mathrm{Ni}>\mathrm{Cu}>\mathrm{Au}$, a similar trend to that recently experimentally confirmed on $\mathrm{CO}_{2}$ hydrogenation on other TMC [7], and, therefore, transferrable to the (001) surface of $\delta$-MoC.

For all the coverages studied $E_{a d s}(\mathrm{Ni})>E_{a d s}(\mathrm{Cu})>E_{a d s}(\mathrm{Au})$. Adsorption causes a charge transfer $\mathrm{MoC} \rightarrow$ adatom in the case of $\mathrm{Au}$ and adatom $\rightarrow \mathrm{MoC}$ for $\mathrm{Cu}$ and $\mathrm{Ni}$, but higher coverage affects all adsorbates quenching electronic charges. This result highlights how electron-electron repulsion governs lateral interactions of the metal adatoms. Finally, adsorption of all considered metals dramatically lowers the work function of the material passing from $\sim 7.5 \mathrm{eV}$ to $\sim 4 \mathrm{eV}$, with concomitant enhancement of its usage in electrocatalysis.

\section{Acknowledgements}

This work was supported by the Spanish MICINN and MINECO (FIS2008-02238 and CTQ201230751 grants, respectively) and by Generalitat de Catalunya (grants 2009SGR1041 and XRQTC). G.G.A. thanks the Universitat Rovira $i$ Virgili for supporting his predoctoral research. F.V. thanks the MINECO for a postdoctoral Juan de la Cierva grant (JCI-2010-06372). F.I. acknowledges additional support through the ICREA Academia award for excellence in research. J.A.R. is grateful for financial support by the US Department of Energy, Basic Energy Sciences Office (DE-AC0298CH10886). Computational time at the MARENOSTRUM supercomputer has been generously provided by the Barcelona Supercomputing Centre. 
Figure 1. Top view of the optimised (001) surface of $\delta$-MoC. Yellow lines indicate the vectors of the supercell models used to study adsorption at various coverage $\theta$. The $(\sqrt{2} \times \sqrt{2}) \mathrm{R} 45^{\circ}$ cell, $(1 \times 1)$ in our notation, exposes 4 atoms in total, $2 \mathrm{Mo}$ and $2 \mathrm{C}$ and represents a coverage $\theta=0.25 .(2 \times 2)$ and (3×3) supercells represent $\theta=0.0625$ and $\theta=0.028$ and expose 16 and 36 atoms respectively. Mo and $\mathrm{C}$ atoms are represented by cyan and purple spheres, respectively.

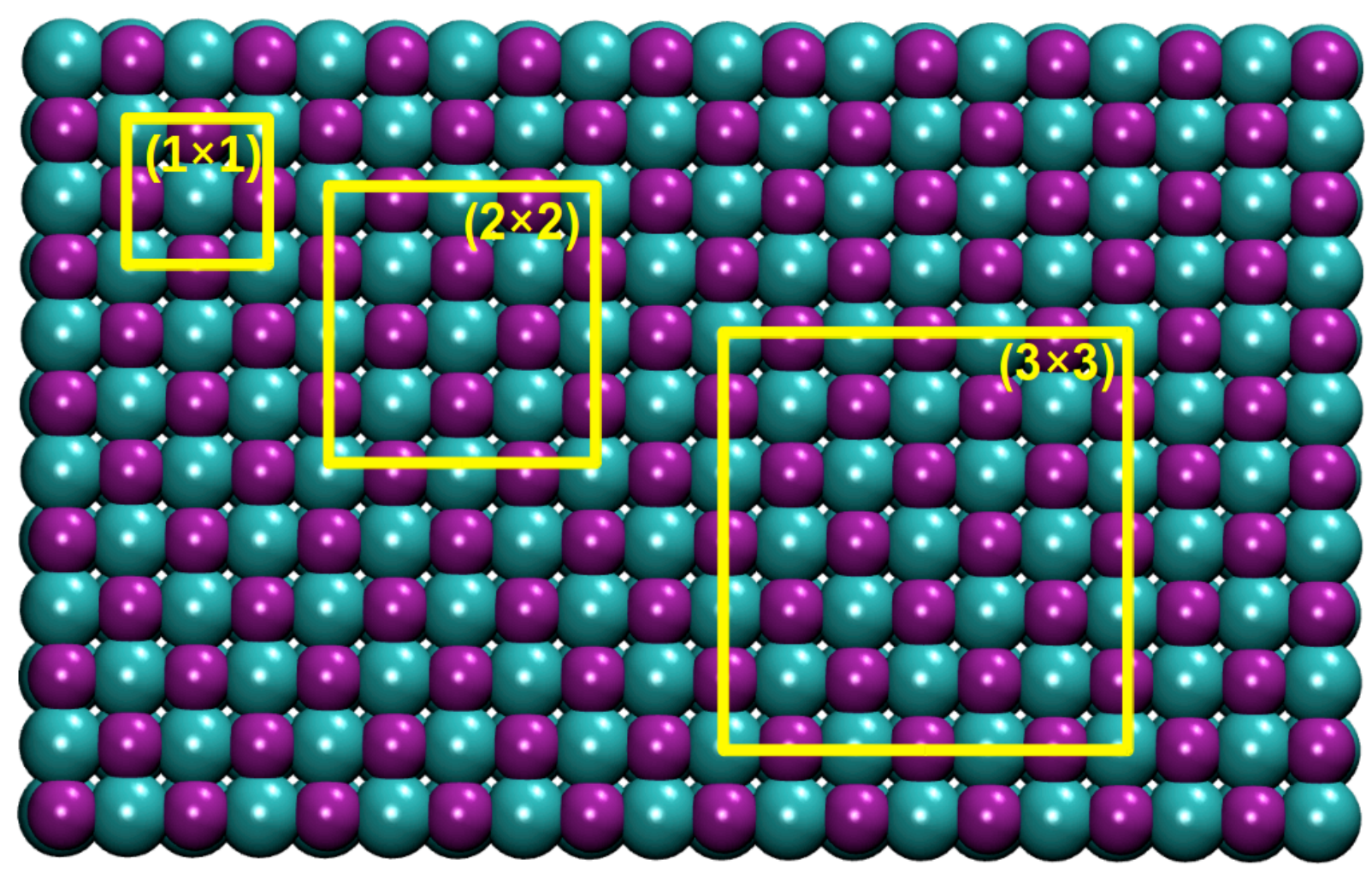


Figure 2. Scatter diagram for calculated adsorption energies versus adsorption areas for each adsorbed metal atom in its most stable adsorption geometry. Vertical dashed line indicates the area of the relaxed naked surface. Graphical explanation of the adsorption area $(A)$, in orange, and perpendicular distance from the surface ( $h$, see Table 1$)$ is reported in the inset for Ni atom, denoted by a grey sphere. Values for adsorption energies and areas are in $\mathrm{eV}$ and $\AA^{2}$ respectively.

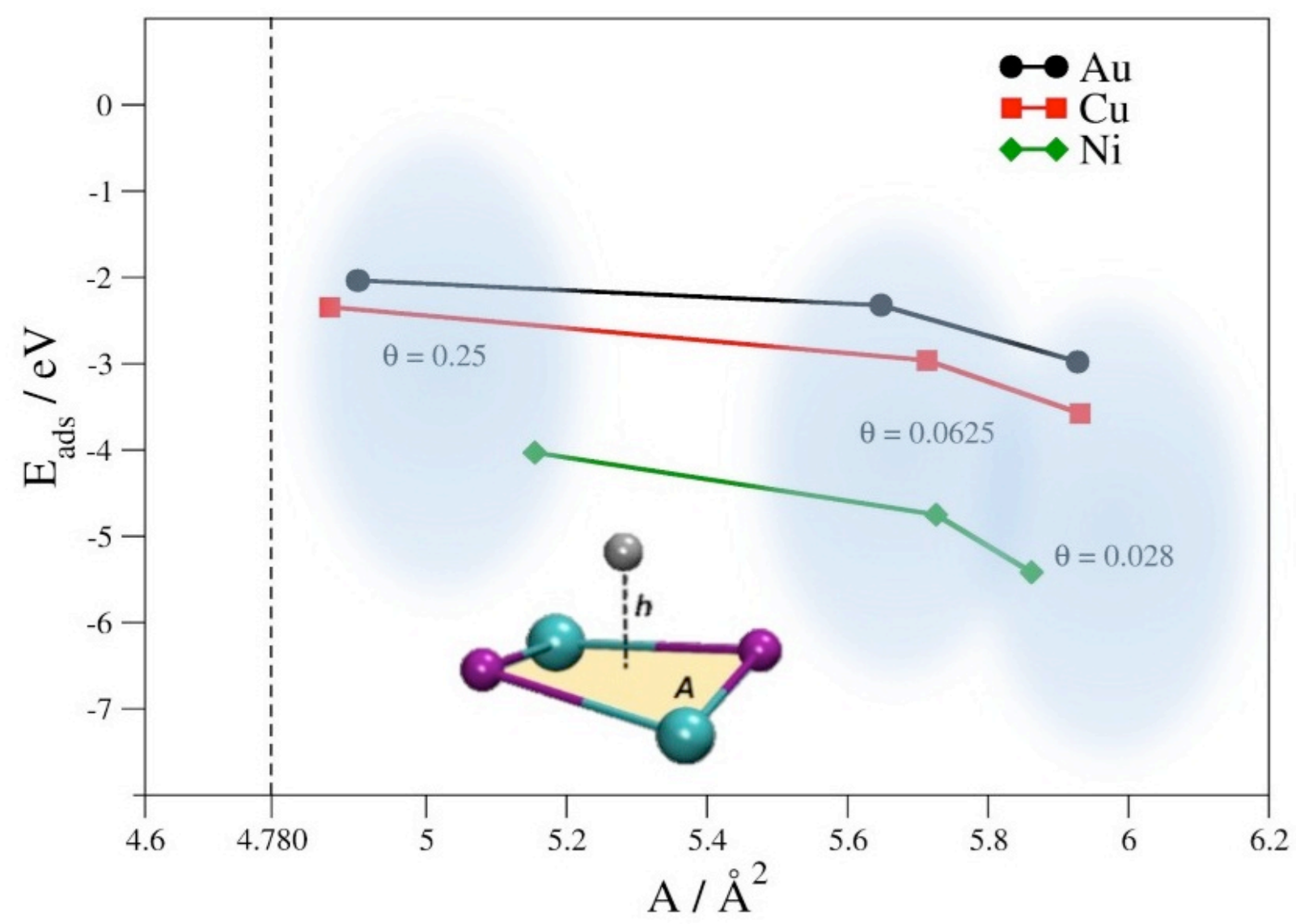


Figure 3. Total DOS and PDOS at each coverage represented for each adatom adsorbed on its most stable geometry. Vertical coloured lines represent the centre of the $d$-band for each coverage, and corresponding values are also reported nearby, in $\mathrm{eV}$. On the left of each panel, eagle-eye view of the optimised structures at low coverage is also reported. $\mathrm{Au}, \mathrm{Cu}$, and $\mathrm{Ni}$ atoms are denoted as orange, red, and grey spheres, respectively.

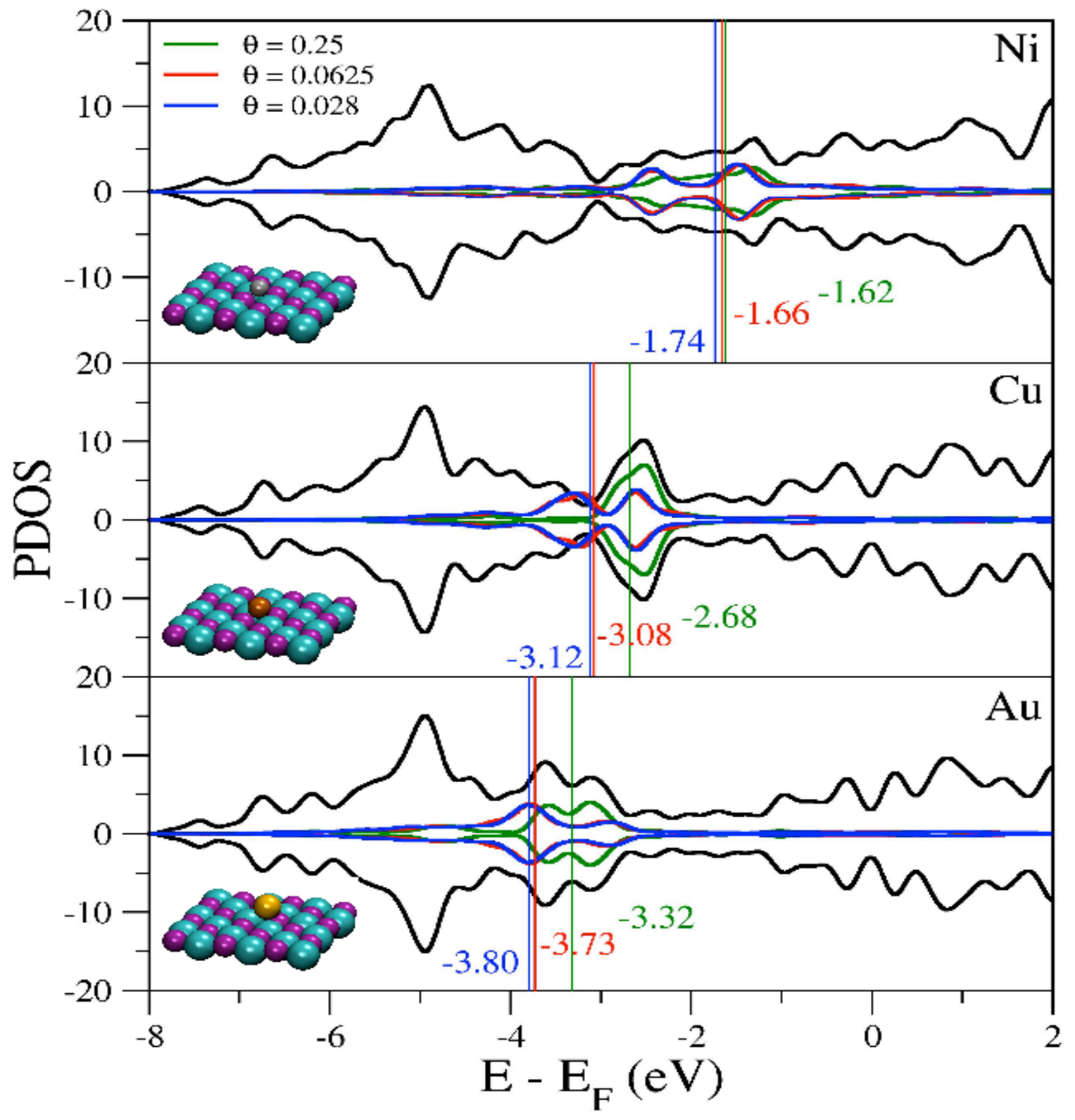


Table 1. Summary of surface properties for $\mathrm{Au}, \mathrm{Cu}$, and $\mathrm{Ni}$ adatoms adsorbed on the (001) surface of $\delta$-MoC at different coverages: Adsorption energies $\left(E_{a d s}\right)$ and work functions $(\phi)$ are reported in $\mathrm{eV}$; perpendicular distance to the surface $(h)$ and adsorption area $(A)$ are given in $\AA$ and $\AA^{2}$, respectively. Atomic charges $(Q)$ are reported in atomic units. Values in italics correspond to the MMC adsorption geometry, while regular font corresponds to Hollow sites. Graphical explanation of adsorption area $(A)$ and perpendicular distance $(h)$ is reported in the inset of Figure 2 for the particular case of $\mathrm{Ni}$.

\begin{tabular}{|c|c|c|c|c|c|c|c|c|c|}
\hline \multirow[t]{2}{*}{$\theta$} & \multicolumn{3}{|c|}{0.028} & \multicolumn{3}{|c|}{0.0625} & \multicolumn{3}{|c|}{0.25} \\
\hline & $\mathbf{N i}$ & $\mathbf{C u}$ & $\mathbf{A u}$ & $\mathbf{N i}$ & $\mathbf{C u}$ & $\mathbf{A u}$ & $\mathbf{N i}$ & $\mathbf{C u}$ & $\mathbf{A u}$ \\
\hline$E_{a d s} / \mathrm{eV}$ & -5.42 & -3.58 & -2.98 & -4.75 & -2.96 & -2.32 & -4.03 & -2.35 & -2.04 \\
\hline$h / \AA$ & 1.32 & 1.47 & 1.98 & 1.34 & 1.51 & 1.98 & 1.491 & 1.83 & 2.21 \\
\hline$A / \AA^{2}$ & 5.86 & 5.93 & 5.93 & 5.73 & 5.71 & 5.65 & 5.16 & 4.86 & 4.90 \\
\hline$Q / \mathrm{e}$ & 0.19 & 0.22 & -0.18 & 0.21 & 0.23 & -0.21 & 0.24 & 0.19 & -0.13 \\
\hline$\phi / e V$ & 4.00 & 3.98 & 3.97 & 3.93 & 3.89 & 3.92 & 3.43 & 3.56 & 3.88 \\
\hline
\end{tabular}




\section{References}

${ }^{1}$ J.A. Rodriguez, F. Illas, Phys. Chem. Chem. Phys. 14 (2012) 427-438.

${ }^{2}$ T. G. Kelly, J. G. Chen, Chem. Soc. Rev. 41 (2012) 8021-8034.

${ }^{3}$ F. Viñes, C. Sousa, P. Liu, J.A. Rodriguez, F. Illas, J. Chem. Phys. 122 (2005) 174709-174720.

${ }^{4}$ F. Viñes, J.A. Rodriguez, P. Liu, F. Illas, J. Catal. 260 (2008) 103-112.

${ }^{5}$ A.L. Stottlemyer, T.G. Kelly, Q. Meng, J.G. Chen, Surf. Sci. Rep. 67 (2012) 201-232.

${ }^{6}$ J.A. Rodriguez, P. Liu, F. Viñes, F. Illas, Y. Takahashi, K. Nakamura, Angew. Chem. Int. Ed. 120 (2008) 6787-6791.

${ }^{7}$ J.A. Rodriguez, J. Evans, L. Feria, A.B. Vidal, P. Liu, K. Nakamura, F. Illas, J. Catal. 307 (2013) 162-169.

${ }^{8}$ F. Viñes, C. Sousa, F. Illas, P. Liu, J.A. Rodriguez, J. Phys. Chem. C 111 (2007) 16982-16989.

${ }^{9}$ Y.F. Zhang, F. Viñes, Y. J. Xu, Y. Li, J. Q. Li, F. Illas, J. Phys. Chem. B 110 (2006) 1545415458.

${ }^{10}$ J.A. Rodriguez, F. Viñes, F. Illas, P. Liu, Y. Takahashi, K. Nakamura, J. Chem. Phys.127 (2007) 211102-211104.

${ }^{11}$ E. Florez, F. Viñes, J.A. Rodriguez, F. Illas, J. Chem. Phys. 130 (2009) 244706-244713.

${ }^{12}$ E. Florez, L. Feria, F. Viñes, J.A. Rodriguez, F. Illas, J. Phys. Chem. C 113 (2009) 19994-20001.

${ }^{13}$ T. Gomez, E. Florez, J.A. Rodriguez, F. Illas, J. Phys. Chem. C. 114 (2010) 1622-1626.

${ }^{14}$ E. Florez, T. Gomez, J.A. Rodriguez, F. Illas, Phys. Chem. Chem. Phys.13 (2011) 6865-6871.

${ }^{15}$ T. Gomez, E. Florez, J.A. Rodriguez, F. Illas, J. Phys. Chem. C, 115 (2011) 11666-11672.

${ }^{16}$ J.A. Rodriguez, L. Feria, T. Jirsak, Y. Takahashi, K. Nakamura, F. Illas, J. Am. Chem. Soc.132 (2010) 3177-3186.

${ }^{17}$ E. Florez, T. Gomez, P. Liu, J.A. Rodriguez, F. Illas, ChemCatChem 2 (2010) 1219-1222.

${ }^{18}$ L.K. Ono, B. Roldán-Cuenya, Catal. Lett. 113 (2007) 86-93.

${ }^{19}$ A.B. Vidal, L. Feria, J. Evans, Y. Takahashi, P. Liu, K. Nakamura, F. Illas, J. Phys. Chem. Lett, 3 (2012) 2275-2280.

${ }^{20}$ F. Viñes, A. Vojvodic, F. Abild-Pedersen, F. Illas, J. Phys. Chem. C, 117 (2013) 4168-4171.

${ }^{21}$ J.R. Politi, F. Viñes, J.A. Rodriguez, F. Illas, Phys. Chem. Chem. Phys. 15 (2013) 12617-12625.

22 J.P. Perdew, K. Burke, M. Ernzerhof, Phys. Rev. Lett. 77 (1996) 3865-3868.

${ }^{23}$ G. Kresse, J. Furthmüller, Phys. Rev. B 54 (1996) 11169-11186.

${ }^{24}$ P.E. Blöch, Phys. Rev. B 50 (1994) 17953-17879.

${ }^{25}$ R.F.W. Bader, Atoms in Molecules: A Quantum Theory, Oxford Science, Oxford, UK, 1990.

${ }^{26}$ J. Goniakowski, C. Noguera, Surf. Sci., 323 (1995) 129-141.

${ }^{27}$ S. Sawada, K. Nakamura, J. Phys. C: Solid State Phys. 12 (1979) 1183-1193. 
${ }^{28}$ J. Perdew, J.A. Chevary, S.H. Vosko, K.A. Jackson, M.R. Pederson, D.J. Singh, C. Fiolhais, Phys. Rev. B 46 (1992) 6671-6687.

${ }^{29}$ F. Viñes, C. Sousa, F. Illas, P. Liu, J.A. Rodriguez, J. Phys. Chem. C 111 (2007) 1307-1314.

${ }^{30}$ L.G.M. Pettersson, P.S. Bagus, Phys. Rev. Lett. 56 (1986) 500-503.

${ }^{31}$ A. Michaelides, P. Hu, M.-H. Lee, A. Alavi, D.A. King, Phys. Rev. Lett. 90 (2003) 246103

32 A. Migani, C. Sousa, F. Illas, Surf. Sci., 574 (2005) 297-305

${ }^{33}$ P.S. Bagus, D. Käfer, G. Witte, C. Wöll, Phys. Rev. Lett. 100 (2008) 126101.

${ }^{34}$ D.V. Esposito, S.T. Hunt, Y.C. Kimmel, J.G. Chen, J. Am. Chem. Soc. 134 (2012) 3025-3033.

${ }^{35}$ J.A. Rodriguez, work in progress

${ }^{36}$ P. Liu, J.A. Rodriguez, T. Asakura, J. Gomes, K. Nakamura, J. Phys. Chem. B, 109 (2009) 45754583.

${ }^{37}$ J.A. Rodriguez, J. Dvorak, T. Jirsak, J. Phys. Chem. B, 104 (2000) 11515-11521. 